\title{
NEW TOOLS FOR MALARIA CONTROL - USING THEM WISELY
}

Running title: New tools for malaria control

\section{Brian Greenwood}

Faculty of Infectious and Tropical Diseases, London School of Hygiene \& Tropical Medicine

\section{Correspondence}

Brian Greenwood

Faculty of Infectious and Tropical Diseases

London School of Hygiene \& Tropical Medicine

Keppel St., London WC1E 7HT, UK

Tel: +442072994707

Email: brian.greenwood@1shtm.ac.uk 


\section{Summary}

The incidence of malaria in sub-Saharan Africa is falling and in many countries on the continent the pattern of malaria infection within the country is becoming more heterogeneous. National malaria control programmes need to take this into account and the 'one size fits all' approach to malaria control may no longer be appropriate, with individual approaches being needed in different parts of a country. This applies particularly to decisions on the introduction of new control tools. Recent experience with Seasonal Malaria Chemoprevention and with the RTS,S/AS01 malaria vaccine provides examples of interventions which need to be deployed on a restricted rather than a national basis, taking account of differences in climate and the intensity of malaria infection between regions within a country. Designing, implementing and monitoring more complex national malaria control programmes will require staff skilled in many disciplines, and substantial funding will be needed to sustain these more complex control programmes, even though the burden of the disease is falling.

Key words: malaria, malaria control; Seasonal Malaria Chemoprevention; RTS,S/AS01 malaria vaccine. 


\section{Introduction}

Measuring the burden of malaria is challenging as the clinical features of the infection are non-specific and mimicked by many other infectious diseases and many deaths from malaria occur at home without prior investigation. However, there is a consensus that during the past 15 years both the number of cases of malaria and the number deaths from malaria has fallen by about a half, despite a substantial increase in the population of malaria endemic areas during this period. ${ }^{1}$ This success has been achieved primarily through scaling up of established interventions - insecticide treated bednets (ITNs), indoor residual spraying (IRS) and artemisinin combination therapy (ACT). Modelling suggests that the most important of these interventions has been the widespread deployment of ITNs; it is estimated that about a half of the population at risk for malaria now sleeps under an ITN, and that approximately $70 \%$ of the reduction in burden between 2000 and 2015 can be attributed to deployment of this intervention. ${ }^{2}$

Recent achievements in malaria control are a cause for celebration but the fact remains that in 2015 there were still an estimated 212 [range 148-304] million cases of malaria and 429 [range 235-639] thousand preventable deaths from malaria, a large majority of the latter occurring in African children. ${ }^{1}$ Further reductions in the malaria burden could be achieved through scaling up of currently employed control tools, especially in areas where coverage remains low, and this is a priority. However, in areas where good coverage has been achieved already, this is likely to become increasingly difficult and expensive as it will be necessary to reach populations that are difficult to access and to persuade those resistant to control measures, such as the use of ITNs, to adopt them. Furthermore, both the efficacy of ITNs and of ACTs are threatened by the emergence and spread of resistance to pyrethroid insecticides
Commented [WA1]: Can I suggest to combine the 2 paragraphs 'introduction' and 'the current malaria situation' to one paragraph.

OK - this has been done. 
in Africa $^{3}$ and of artemisinin resistance in southeast Asia. ${ }^{4}$ Thus, new control tools are needed as an additional measure in areas where current control methods, correctly applied, are not achieving full control of the infection, to replace the insecticides and drugs currently in use if resistance renders them ineffective and finally to contribute to elimination of the infection. Some of the control tools not widely in use that are currently available, or may become so in the near to medium term future, are shown in Table 1.

\section{Deciding on the optimal use of additional malaria control tools}

Development of ACTs was an enormous advance because, at the time that they were introduced, they were highly effective against blood stage infections of all five human malaria parasite species across the malaria endemic world and there was no known resistance. Similarly, when first introduced, ITNs were highly effective in nearly all highly malaria endemic areas where the vector mosquitoes feed predominantly at night. Consequently, at this time, national policies for malaria control could be developed which were simple to follow and could be implemented across a country without a need to take into account regional differences in the epidemiology of the infection. This situation no longer applies in many countries so that the additional methods of malaria control becoming available will need to be deployed in a more focused manner to achieve their maximum impact, taking into account a number of variables such as the seasonality of malaria, its intensity and preferences of the population for specific control measures in different parts of a country. Two recent examples of how a rational, focused approach to the deployment of two new malaria control interventions has been developed or is being considered are described in the following two sections of the paper. 


\section{Seasonal malaria chemoprevention}

In many parts of sub-Saharan Africa, malaria transmission is highly seasonal (Figure 1).

There are few data on the incidence of clinical episodes of malaria by month of year in subSaharan Africa but using monthly rainfall as a surrogate for malaria transmission allowed production of a map of the areas where malaria transmission is likely to be highly seasonal. ${ }^{5}$ These areas are found mostly in the Sahel and sub-Sahel region of Africa, although there are also areas with seasonal transmission in southern and eastern Africa. However, in most of the latter areas, the incidence of the infection is low. In contrast, in the areas of the Sahel and sub-Sahel where transmission is highly seasonal, the incidence of malaria remains very high, despite widespread deployment of current control measures. The population of these areas is in the region of 200-300 million, approximately a third of the population of sub-Saharan Africa.

Chemoprevention of different kinds has been used for the prevention of malaria in the endemic population of countries in Africa on many occasions during the past century but it has rarely been implemented on any scale because of concerns over the difficulty of achieving a high level of coverage, cost, the risk of enhancing drug resistance and a potential to impair the development of naturally acquired immunity. Several of these drawbacks would be diminished if antimalarials were given for a limited period rather than throughout the year. This consideration led to the concept of Seasonal Malaria Chemoprevention (SMC) (previously called Intermittent Preventive Treatment in children [IPTc]), an intervention in which young children resident in areas where malaria transmission is highly seasonal are given a full course of an effective antimalarial combination at monthly intervals on three or four occasions during the peak malaria transmission season. A series of trials conducted in 
countries of the Sahel and sub-Sahel showed that this is a highly effective intervention leading to $70-80 \%$ reductions in the incidence of clinical episodes of malaria and severe malaria and a probable reduction in deaths. ${ }^{6}$ Therefore, in 2012, WHO's Malaria Policy Advisory Committee recommended the implementation of SMC for children under the age of five years using sulphadoxine/pyrimethamine (SP) plus amodiaquine (AQ) in countries of the Sahel and sub-Sahel where malaria transmission is highly seasonal. With support from UNITAID to the ACCESS SMC programme, ${ }^{7}$ and financial support from other donors, SMC is now being widely disseminated across countries of the Sahel and it is estimated that around 18 million children will have received SMC in 2016.

Many countries of West Africa span areas of savannah, where malaria transmission of malaria is highly seasonal, and forest areas where it is not. Thus, these countries have had to develop a regional national malaria control programme in which some areas of the country receive the added intervention of SMC whilst others do not. It was initially considered that implementation of such a policy might prove to be politically sensitive but this has not proved to be the case. The principle of implementing regionally different malaria control policies within the same country has thus been established firmly. The SMC experience has been important in showing that national malaria control programmes can establish different malaria control programmes in different regions of the same country based on a sound knowledge of the epidemiology of the infection, with success and without political interference.

\section{The RTS,S/AS01 malaria vaccine}


Development of the RTS,S/AS01 malaria vaccine, the first malaria vaccine to obtain approval from a major regulatory authority (the European Medicines Agency) has taken 30 years. Results from a large phase 3 trial conducted in 11 centres in 7 countries in sub-Saharan Africa showed that three doses of vaccine given to children aged 5-17 months followed by a booster dose 18 months later gave an efficacy of 36\% against clinical attacks of malaria during a four year period of follow-up. ${ }^{8}$ The vaccine was less effective when given with routine Expanded Programme of Immunisation (EPI) vaccine at the ages of 6-14 weeks. ${ }^{8}$ An unexplained increase in meningitis was observed in older, but not in younger children who received the vaccine and there was a suggestion that the proportion of cases of severe malaria due to cerebral malaria was increased, although the overall incidence of severe malaria was reduced in vaccinated children. For these reasons, and because of concerns about the practicability of giving a fourth dose, WHO's SAGE committee did not recommend immediate deployment of the vaccine on a large scale but the conduct of 3-5 large scale pilot implementation studies. ${ }^{9}$ Funding to support three large pilot studies has now been obtained and these are being planned. Where the vaccine might be be most effective if the results of the pilot studies suggest that it should be deployed more generally requires careful consideration.

The efficacy of RTS,S/AS01 in preventing clinical malaria showed some variation between study sites but the vaccine was effective across all sites, independent of the level of malaria transmission at that site. This was an important finding because the potential public health importance of the vaccine depends not only upon its efficacy but primarily upon the number of cases of malaria it would prevent in a particular epidemiological situation, and hence the potential financial savings to the health system that might follow from its deployment. Analysis of the results of the phase 3 trial by site showed that at Kilifi, Kenya, the site with 
the lowest level of transmission, administration of the vaccine to 1,000 children would save approximately 200 cases over a four year period whilst in another area of Kenya (Siaya), vaccination of 1,000 children would save over 6000 cases (Figure 2) $)^{8}$. The implications of Commented [B6]: The same reference describing the these findings for the Kenyan Ministry of Health are obvious. If the Ministry decides to deploy the vaccine in the country following the pilot studies, then a regional policy will be needed in which the vaccine is deployed only in the areas with the highest level of transmission, such as those situated around Lake Victoria, and not in other parts of the country. Other national malaria control programmes will need to make similar decisions based on detailed knowledge of the epidemiology of malaria in different parts of their country. Unless further malaria vaccines being developed have a very high level of efficacy and are very cheap, similar decisions will be need to be taken about their deployment in the future. Experience with the RTS,S/AS01 vaccines provides an example of an intervention for which intensity of infection will be the major factor in determining where it could be deployed most effectively.

\section{Implications of a focused approach to malaria control}

The need for more flexible control programmes has important consequences for national malaria control programmes. National scale-up of ITNs and ACTs requires predominantly experienced logisticians and not scientists. However, the design, implementation and monitoring of more complex programmes based on considerations of local epidemiological, sociological and economic factors requires staff well trained in a variety of disciplines including epidemiology, entomology, parasitology, social sciences and economics. Careful monitoring of new programmes will be essential so that they can be modified rapidly in the face of changes in the epidemiology of the infection brought about by the success of the 
intervention or by other factors, for example a change in the peak age of cases from young to school-aged children. ${ }^{10}$ This will require a well-trained team of scientists. Thus, paradoxically, as malaria control improves and the malaria burden declines, more rather less well trained staff in areas such as entomology will be needed. Achieving the funds to needed to sustain these experienced teams at a time when the burden of malaria is decreasing will be challenging but is essential if the gains made are to be sustained and malaria to be eliminated.

\section{Conflict of interest}

The London School of Hygiene \& Tropical medicine has received financial support from PATH to support evaluation of the RTS,S/AS01 vaccine.

\section{References}

1. World Health Organization. World Malaria Report 2015. Geneva, World Health Organisation, 2016.

2. Bhatt S, Weiss DJ, Cameron E. et al. The impact of malaria control on Plasmodium falciparum in Africa, 2000-2015. Nature 2015; 526; 207-11.

3. Woodrow CJ, White NJ. The clinical impact of artemisinin resistance in Southeast Asia and the potential for future spread. FEMS Microbiol Rev 2016 Sep 8. pii: fuw037. [Epub ahead of print]

4. Hemingway J, Ranson H, Magill A. et al. Averting a malaria disaster: will insecticide resistance derail malaria control? Lancet 2016;387:1785-8. 
5. Wilson AL on behalf of the IPTc Taskforce. A systematic review and meta-analysis of the efficacy and safety of intermittent preventive treatment of malaria in children (IPTc). PloS One 2011; 6: e16976.

6. Cairns M, Roca-Feltrer A, Garske T. et al. Estimating the potential public health impact of seasonal malaria chemoprevention in African children. Nat Commun 2012; 3: 881.

7. www.access-smc.org

8. RTS,S Clinical Trials Partnership. Efficacy and safety of RTS,S/AS01 malaria vaccine with or without a booster dose in infants and children in Africa; final results of a phase 3 trial, individually randomised, controlled trial. Lancet 2015; 386:31-45.

9. World Health Organisation. Malaria vaccine: WHO position paper-January 2016. Wkly Epidemiol Rec 2016; 91: 33-51.

10. Griffin JT, Hollingsworth TD, Reyburn H. et al. Gradual acquisition of immunity to severe malaria with increasing exposure. Proc R Soc B 2015; 282: 20142657.

11. Burt A. heritable strategies for controlling insect vectors of disease. Phil Trans R Soc B $2014 ; 369: 20130432$.

\section{Figure captions}

Figure 1 The seasonality of malaria episodes in Mali (unpublished data from $\mid$ an on-going trial of the impact of adding azithromycin to the antimalarials used for SMC in Burkina Faso and Mali.
Commented [WA9]: Legend is missing for the $\mathrm{Y}$-axis in the figure

Commented [WA10]: I don't understand this.

There are robust data from Ethiopia showing that mass drug administration of azithromycin for control of trachoma significantly reduced overall child mortality. Thus, it might be expected that giving azithromycin (which is also a weak antimalarial) as part of SMC might have a similar effect. This trial is investigating this. 
Figure 2 The number of cases of malaria prevented over a four- year period by vaccination of 1,000 children aged 5-17 months with the malaria vaccine RTS,S/AS01. The different study sites are ranked according to the level of malaria transmission at that site, with the sites with the lowest level of transmission indicated on the left of the figure. The left hand columns in red (R3C) refer to children who received only the first three doses of vaccine, the right hand columns in blue (R3R) to those who received a subsequent booster dose of vaccine. (RTS,S Clinical Trials Partnership. Lancet 2015; 386:31-45).

\section{Table caption}

Table 1 Additional malaria control interventions not currently used widely and/or under development which might be used in a selective way in a national malaria control programme. 
Table 1 Additional malaria control interventions not currently used widely and/or under development which might be used in a selective way in a national malaria control programme.

\section{Vector control}

Larviciding

Repellents

Mosquito traps

Bacterial infection

of mosquitoes

Genetically modified

mosquitoes $^{2}$

\section{Chemoprevention}

Mass drug administration

Transmission blocking drugs

Ivermectin $^{1}$

\section{Vaccination}

Pre-erythrocytic vaccines

Blood stage vaccines

Transmission blocking vaccines

Notes: ${ }^{1}$ Ivermectin, used in mass drug programmes against filarial infections reduces mosquito survival and hence has a malaria transmission effect.

${ }^{2} \mathrm{~A}$ variety of approaches to genetic modification of mosquitoes are being explored including induction of resistance of the mosquito to malaria infection and production of all male mosquitoes. ${ }^{11}$ 
Fig 1.

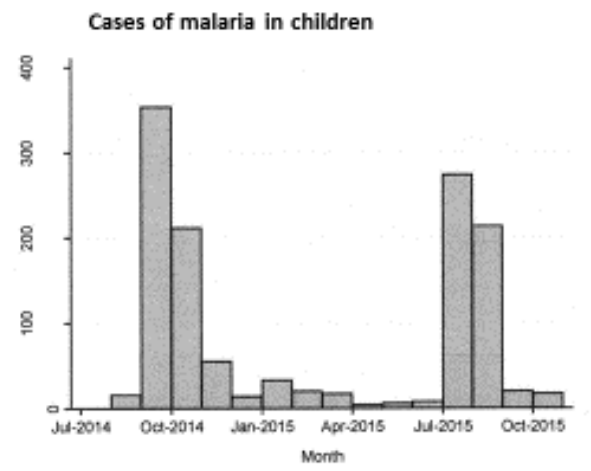

Fig 2

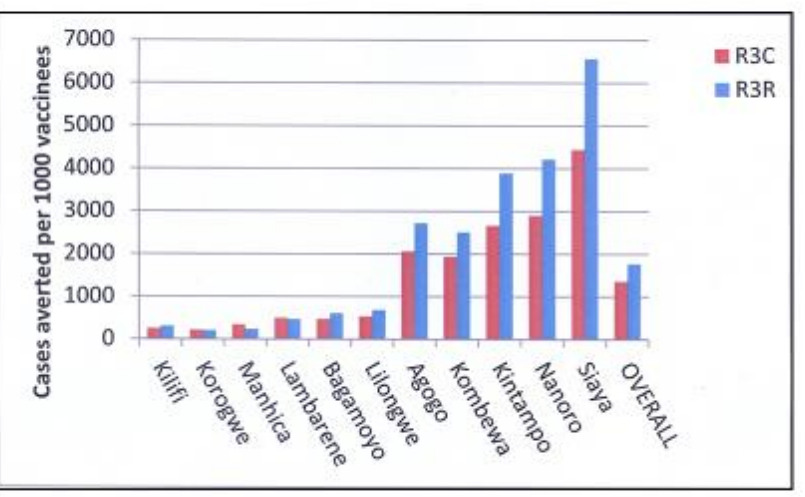

